\title{
Surgery for Triple Pathology of Giant Vestibular Schwannoma Associated with Carotid Artery Stenosis and Intracranial Aneurysm: Case Report
}

\author{
Andriy Sirko $^{1,2}$ Lyudmila Dzyak ${ }^{1}$ Dmytro Romanukha ${ }^{1}$ Grigoriy Pilipenko ${ }^{1,2}$ Yuri Cherednychenko ${ }^{3}$
}

${ }^{1}$ Division of Postgraduate Education, Department of Nervous Diseases and Neurosurgery, Dnipropetrovsk Medical Academy, the Ministry of Health of Ukraine, Dnipro, Ukraine

${ }^{2}$ Cerebral Neurosurgery Department No. 2, Mechnikov Dnipropetrovsk Regional Clinical Hospital, Dnipro, Ukraine

${ }^{3}$ Endovascular Center, Mechnikov Dnipropetrovsk Regional Clinical Hospital, Dnipro, Ukraine

J Neurol Surg Rep 2018;79:e65-e69.

\author{
Address for correspondence Andriy Sirko, MD, PhD, Cerebral \\ Neurosurgery Department No. 2, Mechnikov Dnipropetrovsk Regional \\ Clinical Hospital, 14 Soborna Square, Dnipro, 49005, Ukraine \\ (e-mail: neurosirko75@gmail.com).
}

\begin{abstract} Keywords

- vestibular schwannoma

- carotid artery stenosis

- intracranial aneurysm

- triple pathology

Here we report the case of a 56-year-old woman who presented with a highly unusual combination of three pathologies, namely a giant left-sided vestibular schwannoma, severe stenosis of the left internal carotid artery, and a right anterior cerebral artery aneurysm. The chosen approach comprised three consecutive surgical procedures, namely (1) aneurysm embolization, (2) carotid stenosis stenting, and (3) vestibular schwannoma excision. This approach avoided complications and achieved a satisfactory functional outcome. To the best of our knowledge, this combination of three conditions has not been previously reported in the English-language literature.
\end{abstract}

\section{Introduction}

This report describes a highly unusual case of a woman who presented with three pathologies, namely a giant left-sided vestibular schwannoma (VS), severe stenosis of the left internal carotid artery (ICA), and a right anterior cerebral artery (ACA) aneurysm.

Each individual condition experienced by the patient is relatively rare. The prevalence of cerebral aneurysm in adults has been reported to be 2 to $6 \%,{ }^{1}$ with the prevalence of aneurysmal subarachnoid hemorrhage varying between populations; the highest rates are 22.2 and 32.0 per 100,000 people in Finland and Japan, with a worldwide prevalence of 9.1 per 100,000 people. $^{2}$ The prevalence of ICA atherosclerotic stenosis has been described by four demographic research meta-analyses (MDCS, Tromsø, CAPS, and CHS), which involved 23,706 participants, with the conditions assessed including asymptomatic moderate stenosis (defined as $\geq 50 \%$ ) and severe ICA stenosis (defined as $\geq 70 \%$ ). The prevalence of mild stenosis is reported to be 0.5 to $5.7 \%$ in men and 0.3 to $4.5 \%$ in women, whereas that of severe stenosis to be 0.1 to $1.7 \%$ in men and 0 to $0.9 \%$ in women. ${ }^{3}$ Atherosclerotic extracranial stenosis predominantly localized in ICA bifurcation causes ischemic stroke in $75 \%$ of the cases. ${ }^{4}$ VS account for $8 \%$ of all intracranial tumors and $85 \%$ of cerebellopontine angle tumors, but their annual incidence is $\sim 1$ per 100,000 people. $^{5}$ Two studies have reported the combination of cerebral vessel aneurysms and extracranial main artery stenotic occlusive lesions to be diagnosed in 0.5 to $14.2 \%$ of the cases. ${ }^{6,7}$

The prevalence of concomitant cerebral aneurysm and brain tumor is 0.3 to $7.7 \%{ }^{8,9}$ Aneurysms are most commonly found in combination with meningioma, pituitary adenoma, and glial tumors, although there have been case reports of a combination with VS. ${ }^{10,11}$ A combination of ICA extracranial stenosis and brain tumor is rare and has been described in the literature only as single cases. ${ }^{12}$
License terms

Stuttgart · New York

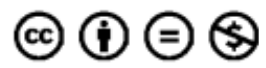

received

October 30, 2017

accepted

May 14, 2018
DOI https://doi.org/

10.1055/s-0038-1666806. ISSN 2193-6358. 
A search of the literature produced no examples of surgical treatment for a combination of VS, ICA extracranial stenosis, and cerebral vessel arterial aneurysm. The PubMed database was searched for English-language articles published from 1970 to 2017 using the keywords brain tumor, VS, carotid artery stenosis, and intracranial aneurysm, in combination with the terms and, combined, associated, or coexistence. This identified only one similar report, which also involved a triple pathology, namely an intradural, infiltrative, and inoperable glomus jugulare tumor, ICA stenosis, and a middle cerebral artery aneurysm. ${ }^{13}$

\section{Case Presentation}

A 56-year-old woman was admitted to our clinic with headache, dizziness, hearing loss in her left ear, dystaxia, unsteady walking, fatigue, rapid fatigability, and occasional weakness in her right limbs. Her medical history showed that $\sim 5$ years previously, she started to notice a gradual progressive hearing loss in her left ear and that she had been suffering from occasional headaches for $\sim 3$ years. She first experienced weakness in her right upper limb 1 year previously, and subsequently noted several further episodes. She received neurological treatment after a transitory ischemic attack, and 4 months before admission, she developed severe headaches, dizziness, and unsteady walking. Magnetic resonance imaging (MRI) revealed a left cerebellopontine angle tumor, following which she was referred to our clinic.

The patient's neurological status included left-sided deafness, right-sided pyramidal insufficiency, and significant coordinative insufficiency. MRI revealed a giant left-sided VS with significant brain stem and fourth ventricle compression; therefore, VS was classified as T4b according to the classification of Samii (-Fig. 1). ${ }^{5}$

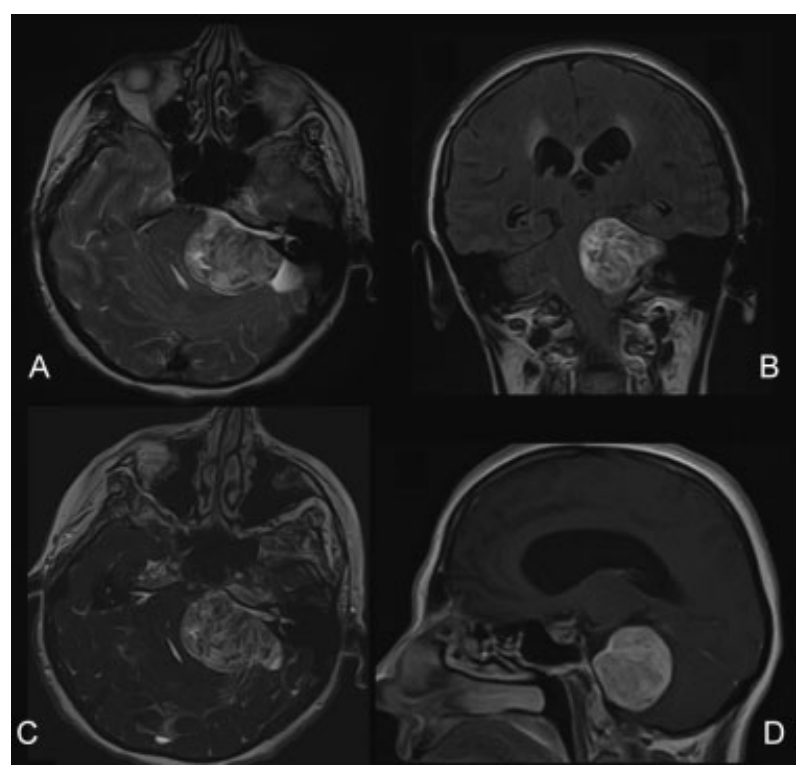

Fig. 1 Pre-surgery magnetic resonance imaging showing a giant left-sided vestibular schwannoma. (A) T2-weighted image. (B) Fluidattenuated inversion recovery image. (C) 3D SSFP IAC. (D) T1 + Gd.

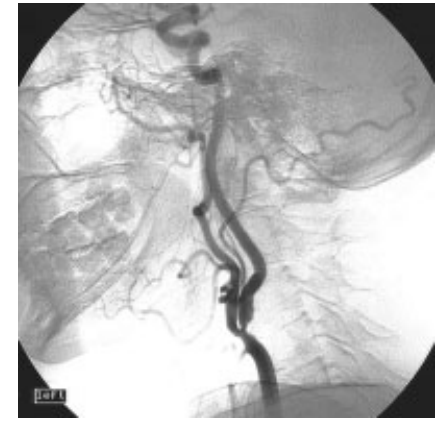

Fig. 2 Carotid angiogram (left side, lateral projection) showing severe internal carotid artery ostium stenosis.

Given her clinical symptoms of cerebral circulatory insufficiency, she was subjected to a thorough preoperative examination, which included Doppler sonography of the main head arteries and selective subtraction digital angiography of cerebral vessels. The Doppler sonography revealed severe left ICA ostial stenosis, which was subsequently confirmed by cerebral angiography (NASCET 85\%) (-Fig. 2). The angiography also revealed a small aneurysm (3-mm diameter) in the corner of A1 to A2 segments of the right ACA (-Fig. 3). Compression tests during cerebral angiography showed an absence of the circle of Willis disintegration.

Thus, a thorough diagnostic examination determined a triple pathology of a giant left-sided VS, subtotal left ICA stenosis, and an aneurysm in the corner of the right ACA A1 to A2 segments. Therefore, a three-stage treatment process was planned and implemented; the detailed reasoning behind this process is presented in the Discussion section.

\section{Surgery Stage I: Endovascular Embolization of the Right ACA Aneurysm}

Using a transfemoral right-sided approach, a Guider Softip XF 6.0-F guiding catheter (Stryker Neurovascular, Kalamazoo, Michigan, United States) was inserted into the patient's right ICA, and an Excelsior-10 microcatheter (Stryker Neurovascular) on a Transend-10 microconductor(Stryker Neurovascular) was inserted into the aneurysm cavity. The aneurysm cavity was embolized with GDS-10 Ultrasoft $3 \mathrm{~mm} \times 4$-cm and $2 \mathrm{~mm} \times 3-\mathrm{cm}$ microspirals (Stryker Neurovascular). The aneurysm was removed from the blood stream while maintaining the passability of the right carotid basin arteries. There were no signs of angiospasm (-Fig. 4). Her clinical status remained unchanged.

\section{Surgery Stage II: Endovascular Stenting of the Left ICA}

Eight days after the aneurysm embolization, a carotid stent was implanted, followed by preliminary double antiplatelet preparation (clopidogrel, $75 \mathrm{mg} /$ day and aspirin, $75 \mathrm{mg} /$ day for 5 days). Using a right-sided femoral approach, a Vista Brite Tip 8.0-F guiding catheter (Cordis Corporation, Baar, Switzerland) was installed in the left ICA. A distal antiembolic device, Angioguard (Cordis Corporation), was inserted and opened behind the stenosis zone in the ICA, and the stenosis was predilated with a Sterling $5.0 \mathrm{~mm} \times 20.0$-mm balloon catheter (Boston Scientific, Marlborough, Massachusetts, United States). 


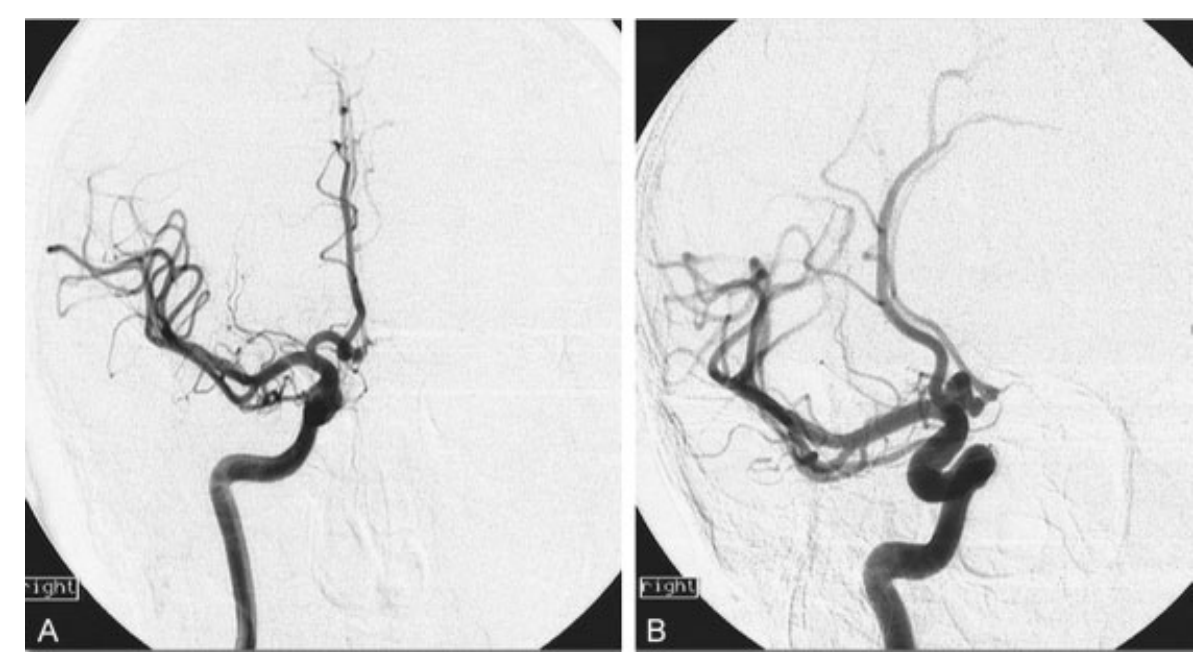

Fig. 3 (A) Carotid angiogram (right side, frontal projection) showing an aneurysm in the A1 to A2 segments of the right anterior cerebral artery. (B) Semi-lateral projection showing an overflow in the anterior communicating artery.
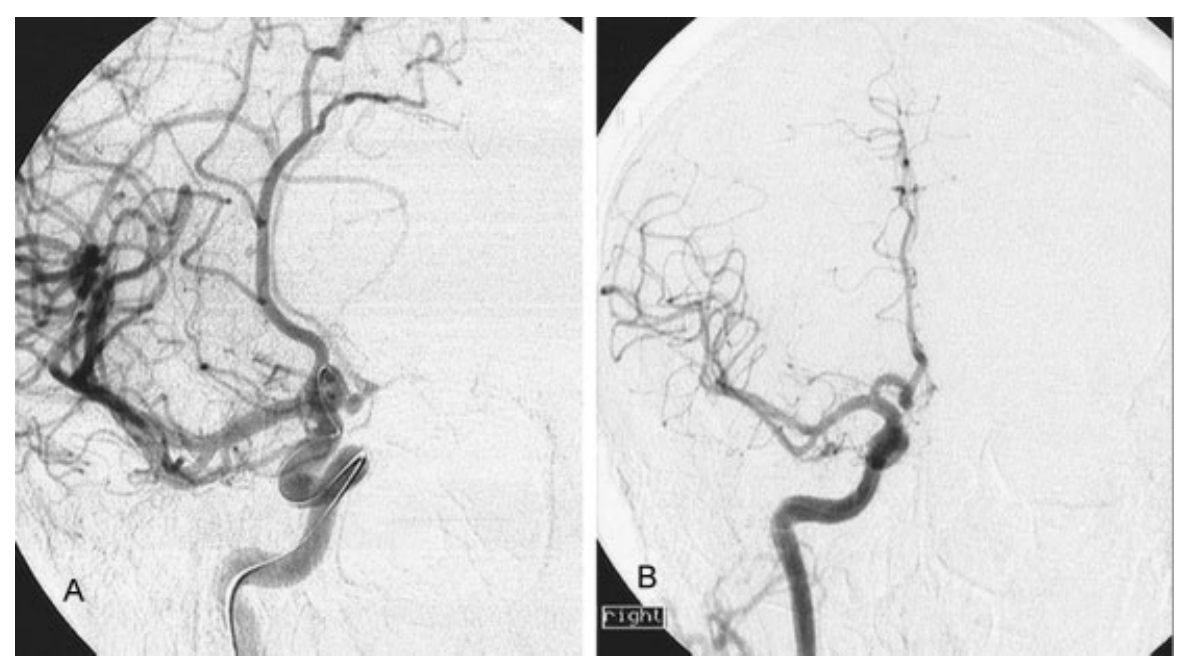

Fig. 4 (A) Intraoperative right-sided carotid angiogram (semi-lateral projection) showing a microcatheter inserted into the aneurysm in the corner of the A1 to A2 segments of the right anterior cerebral artery. (B) Postoperative direct projection with the aneurysm is not contrasted, confirming that the right carotid basin arteries were passable.

A Cristallo Ideale 6 to $9 \mathrm{~mm} \times 30$ - $\mathrm{mm}$ stent (Medtronic, Minneapolis, United States) was implanted in the stenosis zone, and the stenosis was postdilated with a Sterling $6.5 \mathrm{~mm}$ $\times$ 20.0-mm balloon catheter (Boston Scientific). The stenosis was eliminated, and the left carotid basin arteries were left passable (-Fig. 5). The patient's clinical status remained unchanged. After the stenting, she underwent 2 months of double antiplatelet therapy.

\section{Surgery Stage III: Total Excision of the Giant Left-Sided vS}

VS excision surgery was performed 2 months after eliminating the ICA stenosis. Prior to this final stage of surgical treatment, the patient underwent Doppler sonography of the main head arteries for control purposes. This showed that the arteries remained passable, with no restenosis detected.

A retrosigmoid approach to the tumor was used. The lateral cerebellomedullary cistern was opened, and cerebrospinal fluid was evacuated to decompress the tentorium.

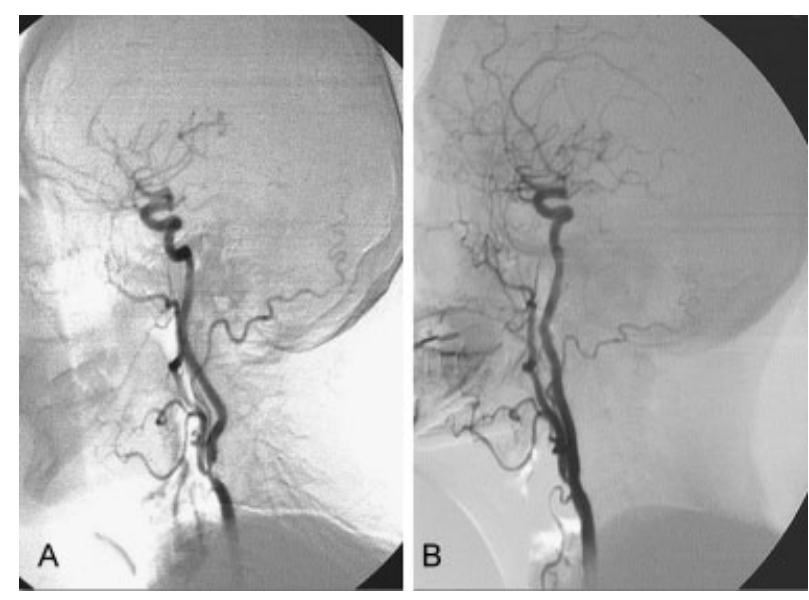

Fig. 5 (A) Preoperative carotid angiogram (left side, lateral projection) showing severe stenosis in the internal carotid artery (ICA) ostium. (B) Postoperative angiogram (lateral projection) showing that the left ICA ostial stenosis had been eliminated and the arteries were passable. 


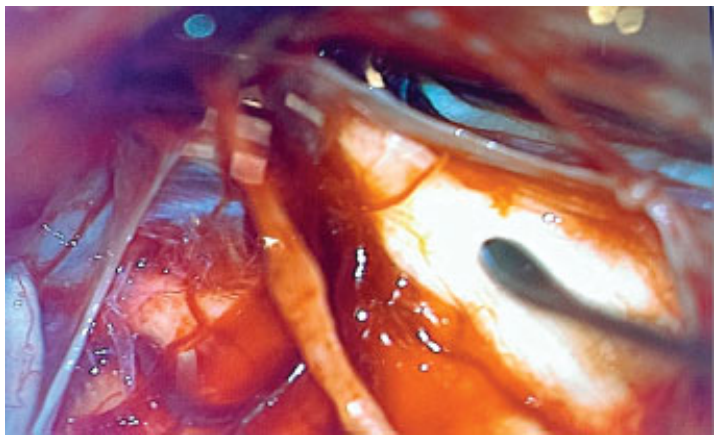

Fig. 6 Intraoperative image confirming the total removal of the giant vestibular schwannoma. Cranial nerves, from left to right: VI, VII, V, and IV. The dissector tip can be seen on the trigeminal nerve.

The intracapsular tumor was removed as much as possible using microsurgical tools under optical magnification with an OPMI VARIO 700 microscope (Carl Zeiss, Oberkochen, Germany) and with continuous neurophysiological monitoring of facial nerve function using a Nim Response 3.0 nerve monitor (Medtronic). The trigeminal nerve, Dandy's vein, and the pathetic nerve were first verified and isolated. The internal auditory canal was then enlarged to $1.5 \times 1.2 \mathrm{~cm}$, and a part of its posterior wall was drilled. The intracanalicular part of the tumor was removed. Using subarachnoid dissection, a large part of the tumor capsule was separated from the pons, leaving the pons and its veins intact. The abducent nerve and basilar artery were also verified. The facial nerve was tightly connected to the tumor capsule, significantly thinned, but it was possible to identify the nerve and maintain its complete integrity (-Fig. 6).

After the procedure, the facial nerve retained its integrity both anatomically and functionally. The patient developed no new neurological disorders or complications postoperatively. Upon her discharge, she underwent a follow-up brain MRI with intravenous enhancement by gadolinium injection. The T1-weighted image confirmed the total removal of the tumor, including its intracanalicular part ( - Fig. 7). During the discharge, her facial nerve function corresponded to grade III of the House-Brackmann classification. By the time of the first follow-up 6 months after the surgery, the function had improved to grade II.

\section{Discussion}

A key challenge in treating this patient's triple pathology was the development of the consecutive step surgical treatment plan. Because the treatment of one disease may limit that of another disease, a vicious circle can occur. Because the giant VS was associated with subtotal ICA stenosis and a cerebral aneurysm, removing it first could have led to both ischemic stroke and aneurysm rupture. Changes in brain hemoperfusion during general anesthesia or because of cerebral blood supply restructuring after tumor removal can increase the risk of ischemic brain damage in the stenotic ICA basin. ${ }^{12}$ Similarly, craniotomy and intracranial pressure change can increase the risk of aneurysm rupture..$^{8-11}$ For these reasons,

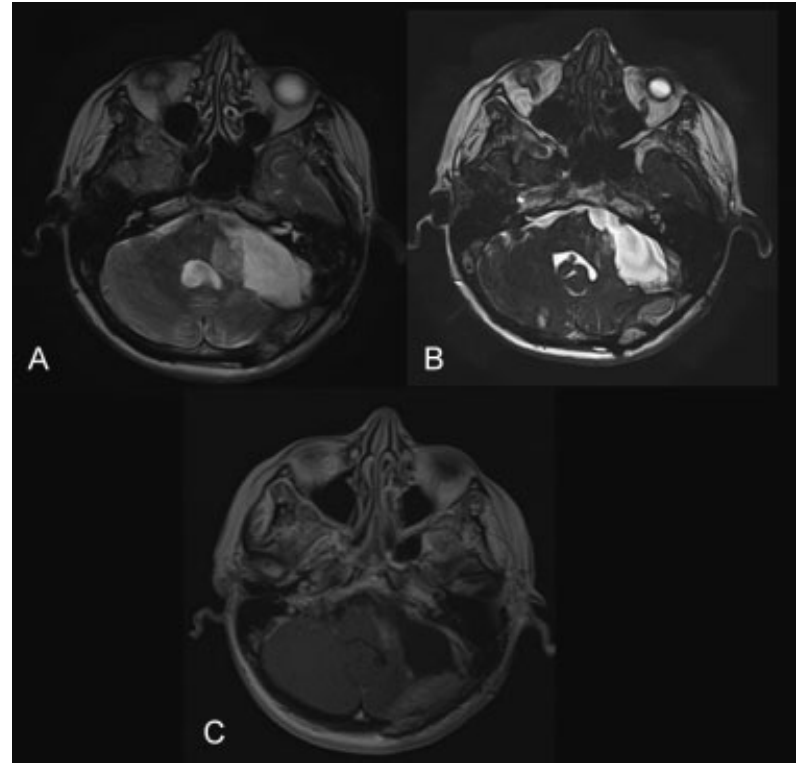

Fig. 7 Brain magnetic resonance imaging after the total removal of left-sided vestibular schwannoma. (A) T2-weighted image. (B) 3D SSFP AIC. (C) T1 + Gd.

the vascular pathology should be initially considered. However, whether the stenosis or the aneurysm should be treated first surgically remains unclear. If stenosis elimination is the first step, then this may increase the risk of aneurysm rupture due to the higher arterial blood flow to cerebral vessels. ${ }^{6,7}$ As the circle of Willis remains intact, the restoration of the left ICA opening may increase the perfusion pressure in both the right carotid basin and adjacent cerebral arterial basins, which, in turn, can increase the risk of aneurysm rupture. Consequently, the first step was to address the aneurysm to prevent the possibility of its rupture during subsequent procedures. The second step was to eliminate the ICA stenosis and restore the normal cerebral blood flow, to minimize the risk of ischemic stroke during the subsequent perioperative period. The final step was to remove the giant VS, which caused brain stem dislocation.

Using endovascular aneurysm embolization rather than transcranial clipping minimized the risk of left ICA stenosis because it did not require general anesthesia or craniotomy, which could have altered cerebral hemoperfusion. We also considered the possibility of performing both carotid stenting and aneurysm embolization in a single session; however, given the need for double antiplatelet preparations prior to carotid stenting, this option was discounted to reduce the risk of bleeding from the aneurysm during the preoperative preparation.

\section{Conclusion}

This patient presented with a highly unusual combination of three pathologies, namely a giant VS, severe stenosis of the left ICA, and a right ACA aneurysm. This required careful consideration to develop a treatment plan that minimized potentially serious risks arising from interactions between 
the procedures. The resultant plan was (1) aneurysm embolization, (2) carotid stenosis stenting, and (3) VS excision. This avoided complications and achieved a satisfactory functional outcome.

\section{References}

1 Mohan D, Munteanu V, Coman T, Ciurea AV. Genetic factors involves in intracranial aneurysms-actualities. J Med Life 2015; 8(03):336-341

2 Tromp G, Weinsheimer S, Ronkainen A, Kuivaniemi H. Molecular basis and genetic predisposition to intracranial aneurysm. Ann Med 2014;46(08):597-606

3 de Weerd M, Greving JP, Hedblad B, et al. Prevalence of asymptomatic carotid artery stenosis in the general population: an individual participant data meta-analysis. Stroke 2010;41(06): 1294-1297

4 Park JH, Razuk A, Saad PF, et al. Carotid stenosis: what is the highrisk population? Clinics (Sao Paulo) 2012;67(08):865-870

5 Samii M, Gerganov V. Surgery of Cerebellopontine Lesions. Heidelberg: Springer-Verlag; 2013:885

6 Borkon MJ, Hoang H, Rockman C, et al. Concomitant unruptured intracranial aneurysms and carotid artery stenosis: an institutional review of patients undergoing carotid revascularization. Ann Vasc Surg 2014;28(01):102-107
7 Cho YD, Jung KH, Roh JK, Kang HS, Han MH, Lim JW. Characteristics of intracranial aneurysms associated with extracranial carotid artery disease in South Korea. Clin Neurol Neurosurg 2013;115(09):1677-1681

8 Javalkar V, Guthikonda B, Vannemreddy P, Nanda A. Association of meningioma and intracranial aneurysm: report of five cases and review of literature. Neurol India 2009;57(06):772-776

$9 \mathrm{Kim} \mathrm{YH,} \mathrm{Lee} \mathrm{YJ,} \mathrm{Han} \mathrm{JH,} \mathrm{et} \mathrm{al.} \mathrm{Association} \mathrm{of} \mathrm{intracranial} \mathrm{aneur-}$ ysms and meningiomas: a case-control study. J Neurosurg 2015; 123(02):357-361

10 Spitler K, Drazin D, Hanna G, Patel A, Chu R. Association of intracranial aneurysms with meningiomas, pituitary adenomas, and gliomas: review of possible interrelationships [full text]. ISRN Neurol [serial online]. 2013;2013:383425. Available at: https:// www.hindawi.com/journals/isrn/2013/383425/. Accessed June 26 , 2013

11 Park KY, Kim BM, Kim DJ. Preoperative coiling of coexisting intracranial aneurysm and subsequent brain tumor surgery. Korean J Radiol 2016;17(06):931-939

12 Xu Z, Luo B, Wang Q, Peng Z, Liang H. Glioblastoma multiform with ipsilateral carotid artery stenosis: carotid artery stent promote tumor growth? [full text]World J Surg Oncol 2016;14(01):32 https://wjso.biomedcentral.com/articles/10.1186/s12957-016-07 82-z AccessedJanuary262016 [serial online]

13 Oláh C, Czabajszki M, Lázár I. [Endovascular treatment of complex vascular pathology in neck and brain region. Case report] [Article in Hungarian]. Orv Hetil 2017;158(18):706-710 